\title{
Seleção de isolados de Paecilomyces lilacinus (Thom.) Samson para controle de Meloidogyne paranaensis em tomateiro
}

\author{
Selection of isolates of Paecilomyces lilacinus (Thom.) Samson to control Meloidogyne paranaensis in tomato
}

\author{
Débora Cristina Santiago $^{1 *}$ Martin Homechin ${ }^{1}$ João Flávio Veloso Silva ${ }^{2}$ Emerson Rodrigo Ribeiro ${ }^{1}$ \\ Bruno Caetano Gomes ${ }^{1}$ Patrícia Helena Santoro ${ }^{1}$
}

\section{RESUMO}

\begin{abstract}
Paecilomyces lilacinus é uma espécie fúngica utilizada no controle biológico de nematóides, sendo uma das mais estudadas a campo. O trabalho teve como objetivo selecionar isolados de Paecilomyces lilacinus quanto à capacidade de controlar Meloidogyne paranaensis em tomateiro cv. "Santa Clara”, em de casa-de-vegetação. Plantas cultivadas em vasos foram inoculadas com M. paranaensis. Após 45 dias da inoculação, efetuou-se a substituição dos tomateiros e foram aplicados 50 gramas de arroz autoclavado e colonizado por P. lilacinus. Decorridos 45 dias da inoculação do fungo, foram avaliados: número de galhas, massa de ovos, número de ovos por sistema radicular, peso da massa fresca da parte aérea, do sistema radicular e sobrevivência do $\boldsymbol{P}$. lilacinus no solo. Todos os tratamentos com incorporação de P. lilacinus reduziram a população de $\boldsymbol{M}$. paranaensis em raízes de tomateiro. Os isolados UEL pae 05, 08, 09, 13, 20, 21, 38, 41, 44, 54, ESALQ 831 e 832 foram os melhores no controle de $\boldsymbol{M}$. paranaensis, afetando a reprodução dos nematóides $e$ apresentando elevada sobrevivência no solo.
\end{abstract}

Palavras-chave: nematóide de galhas, controle biológico, parasita de ovos.

\section{ABSTRACT}

Paecilomyces lilacinus is a fungal species used in biological control of nematodes. It is considered one of the most frequently evaluated fungus under field conditions. The present study aimed to select $\boldsymbol{P}$. lilacinus isolates with regard to their capacity in controlling Meloidogyne paranaensis in tomato. Santa Clara tomato plants were grown in pots and inoculated with M. paranaensis under greenhouse conditions. The tomato plants were replaced 45 days after inoculation with 50 grams of autoclaved rice colonized with P. lilacinus. Fortyfive days after fungus inoculation the number of galls, egg masses, number of eggs in root system, aerial part mass weight, root mass weight and P. lilacinus survival in the soil were evaluated. All treatments involving $\boldsymbol{P}$. lilacinus incorporation reduced the population of $\boldsymbol{M}$. paranaensis in tomato roots. UEL pae 05, 08, 09, 13, 20, 21, 38, 41, 44, 54, ESALQ 831 e 832 were the best in the control of $M$. paranaensis, affecting reproduction of the nematodes and showing a high survival rate in the soil.

Key words: gall nematode, biological control, egg parasite.

\section{INTRODUÇÃO}

A pressão da sociedade no sentido de substituir os nematicidas por produtos ou técnicas ecologicamente mais recomendáveis tem incentivado a busca de métodos alternativos para o controle de fitonematóides (FERRAZ \& SANTOS, 1995), principalmente para os gêneros de maior importância, como Meloidogyne Göeldi. Nesse contexto, o controle biológico vem sendo considerado uma das alternativas dentro de uma abordagem integrada, no qual se busca assegurar o desenvolvimento sustentável da agricultura. $\mathrm{O}$ uso de inimigos naturais é promissor e torna-se um fascinante campo de investigação, sendo potencialmente útil dentro das medidas duráveis (STIRLING, 1991) e podendo atuar reduzindo populações de fitonematóides para limiares abaixo do nível de dano econômico (DUNCAN, 1991).

\footnotetext{
${ }^{1}$ Departamento de Agronomia, Universidade Estadual de Londrina (UEL), CP 6001, 86051-990, Londrina, PR, Brasil. E-mail: santiago@uel.br. *Autor para correspondência.

${ }^{2}$ Empresa Brasileira de Pesquisa Agropecuária, Embrapa-Soja, CP 231, 86001-970, Londrina, PR, Brasil. E-mail: veloso@cnpso.embrapa.br.
} 
Paecilomyces lilacinus (Thom.) Samson é um fungo do solo que tem se mostrado efetivo no biocontrole de espécies de Meloidogyne (KERRY, 1990). É um hifomiceto da ordem Moniliales distribuído por todo o mundo, com maior freqüência em regiões quentes (CARNEIRO, 1986). Caracteriza-se por penetrar nos ovos dos nematóides, destruindo o embrião, podendo exercer forte pressão na capacidade reprodutiva das fêmeas que são colonizadas e posteriormente mortas (DUNN et al., 1982). No Brasil, existem registros de $\boldsymbol{P}$. lilacinus em diferentes tipos de solo, cultivados ou não, em profundidades variáveis de $0-40 \mathrm{~cm}$ ou mais (CARNEIRO, 1986). Freqüentemente tem sido isolado a partir de diferentes hospedeiros ou de substratos provenientes de várias localidades (FARIA\& TIGANO, 1996; SOSA-GOMEZ, 2002), com distribuição cosmopolita e maior freqüência em solos agricultáveis (DOMSCH et al., 1980). A presença de $\boldsymbol{P}$. lilacinus e outros parasitos de ovos foi detectada em solos cultivados com pessegueiro e quiabeiro nos municípios de Pelotas - RS e Atalaia - PR (SANTOS et al., 1991). TIGANO-MILANI et al. (1993) encontraram 29 isolados de $\boldsymbol{P}$. lilacinus em amostras de solo oriundas de 22 municípios distribuídos nos estados da BA, GO, MA, MG, MS, MT, RS, SP e TO. COSTA et al. (1997), estudando a associação de fungos a cistos de Heterodera glycines, encontraram $\boldsymbol{P}$. lilacinus nos municípios de Iraí de Minas - MG e Chapadão do Céu -GO.

Estudos envolvendo a seleção de isolados de $\boldsymbol{P}$. lilacinus para o controle de nematóides são importantes na busca de microrganismos eficientes, adaptados às diferentes regiões. CABANILLAS et al. (1989a), avaliando 13 isolados de P. lilacinus provenientes do Peru e de diversos locais dos Estados Unidos e do Japão, observaram que um originário do Peru e uma mistura dos isolados da Flórida, de Nova York, da Califórnia e do Peru reduziram significativamente o número de galhas formadas por M. incognita em tomateiro. FREITAS et al. (1995), comparando a eficiência do parasitismo de 19 isolados de $\boldsymbol{P}$. lilacinus, observaram que $100 \%$ dos ovos de $\boldsymbol{M}$. javanica estavam parasitados com os isolados originários da Itália e do Peru e cerca de 70\% com o isolado da França. Já o percentual de ovos parasitados pelos isolados brasileiros variou de 2 a $69 \%$. Posteriormente, FREITAS et al. (1999) obtiveram sucesso na proteção de mudas de tomateiro, em casade-vegetação, contra $\boldsymbol{M}$. javanica, por meio da incorporação de $\boldsymbol{P}$. lilacinus ao substrato. Entretanto, NOVARETTI et al. (1986), na cultura de cana-de-açúcar, HEWLETT et al. (1988), em tabaco, e CARNEIRO \& CAYROL (1991), em tomateiro, contestaram a eficiência de diferentes isolados do P. lilacinus como agentes de controle em condições de campo. Resultados contraditórios podem estar relacionados à inadequação dos métodos de aplicação e avaliação (KERRY, 1990), à não-adaptação do isolado a diferentes condições e tipos de solo (CARNEIRO, 1992) e à inadequação dos métodos de produção de conídios do fungo (KERRY, 1990).

Embora resultados encorajadores sejam observados em condições brasileiras (CARNEIRO \& GOMES, 1993; COSTA \& CAMPOS, 1997; FREITAS et al., 1999; MIZOBUTSI et al., 2000), informações básicas sobre o comportamento de $\boldsymbol{P}$. lilacinus como parasita de nematóides de galhas são necessárias para que seu emprego na agricultura seja recomendado, especialmente no controle de $\boldsymbol{M}$. paranaensis, nematóide carente de informações sob o seu manejo. Assim, o presente estudo teve como objetivo avaliar a capacidade apresentada por isolados brasileiros de $\boldsymbol{P}$. lilacinus, obtidos de diferentes locais e substratos, para controlar M. paranaensis em tomateiro.

\section{MATERIAL E MÉTODOS}

O experimento foi conduzido em casa-devegetação, em delineamento inteiramente casualizado, tendo sido avaliados 38 tratamentos, compreendidos por 37 isolados de $\boldsymbol{P}$. lilacinus (Tabela 1) e por uma testemunha sem aplicação do fungo. Deste isolados, 31 foram obtidos pela Universidade Estadual de Londrina (UEL), oriundos de amostras de solos de diversas culturas, pastagens e matas, coletados nos Estados do Paraná, São Paulo, Rio Grande do Sul e Mato Grosso do Sul, dois isolados fornecidos pela ESALQ(831 e 832) e quatro (CG 266, CG 402, CG 184 e CG 175) cedidos pela EMBRAPA - Cenargen.

Os isolados de $\boldsymbol{P}$. lilacinus da UEL foram obtidos por meio da técnica de diluição seriada dos solos e do plaqueamento em meio de cultura semiseletivo de ALVES et al. (1998) modificado, com $20 \mathrm{~g}$ de farinha de aveia, $20 \mathrm{~g}$ de ágar, 300mg de Venturol (dodine

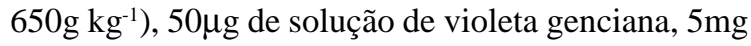
de Tetraciclina (Cloridrato de tetraciclina, 300mg), diluídos em 1000mL de água destilada. Após a obtenção e a identificação dos isolados de $\boldsymbol{P}$. lilacinus, estes foram repicados para tubos de ensaio contendo meio BDA (batata dextrose ágar) e armazenados em geladeira.

Para multiplicação de inóculo dos isolados de $\boldsymbol{P}$. lilacinus, foram utilizados sacos de polipropileno contendo $200 \mathrm{~g}$ de arroz descascado e pré-cozido, autoclavados a $120^{\circ} \mathrm{C}$ durante 20 minutos. Após o resfriamento, cada embalagem foi inoculada com três discos de $8 \mathrm{~mm}$ de diâmetro retirados das colônias fúngicas, contendo micélio e esporos, desenvolvidas 
Tabela 1 - Origem dos isolados de Paecilomyces lilacinus utilizados no estudo.

\begin{tabular}{|c|c|c|}
\hline Código acesso & Hospedeiro ou substrato & Município / Estado \\
\hline UEL pae 01 & Solo (mata nativa) & Cambé, PR \\
\hline UEL pae 02 & Solo (café) & Cambé, PR \\
\hline UEL pae 03 & Solo (trigo) & Cambé, PR \\
\hline UEL pae 05 & Solo (mata nativa) & Rolândia, PR \\
\hline UEL pae 06 & Solo (pastagem) & Rolândia, PR \\
\hline UEL pae 08 & Solo (trigo) & Rolândia, PR \\
\hline UEL pae 09 & Solo (milho) & Rolândia, PR \\
\hline UEL pae 12 & Solo (Pinus sp.) & São Martinho, PR \\
\hline UEL pae 13 & Solo (pastagem) & São Martinho, PR \\
\hline UEL pae 15 & Solo (milho) & Prado Ferreira, PR \\
\hline UEL pae 17 & Solo (pastagem) & Prado Ferreira, PR \\
\hline UEL pae 18 & Solo (pastagem) & Florestópolis, PR \\
\hline UEL pae 19 & Solo (cana-de-açúcar) & Florestópolis, PR \\
\hline UEL pae 20 & Solo (milho) & Florestópolis, PR \\
\hline UEL pae 21 & Solo (trigo) & Florestópolis, PR \\
\hline UEL pae 22 & Solo (mata nativa) & Florestópolis, PR \\
\hline UEL pae 23 & Solo (café) & Alvorada do Sul, PR \\
\hline UEL pae 24 & Solo (trigo) & Alvorada do Sul, PR \\
\hline UEL pae 38 & Solo (quiabeiro) & Distrito Espírito Santo, Londrina, PR \\
\hline UEL pae 39 & Solo (milho) & Bela Vista do Paraíso, PR \\
\hline UEL pae 41 & Solo (pastagem) & Bela Vista do Paraíso, PR \\
\hline UEL pae 42 & Solo (café) & Bela Vista do Paraíso, PR \\
\hline UEL pae 44 & Solo (amora) & Cambé, PR \\
\hline UEL pae 45 & Solo (milho) & Cambé, PR \\
\hline UEL pae 47 & Solo (café) & Cambé, PR \\
\hline UEL pae 48 & Solo (trigo) & Cambé, PR \\
\hline UEL pae 49 & M. javanica e H. glycines raça 3 & Sonora, MS \\
\hline UEL pae 51 & M. incognita e $H$. glycines & Cornélio Procópio, PR \\
\hline UEL pae 52 & M. incognita e $H$. glycines & Forínea, SP \\
\hline UEL pae 54 & H. glycines & Marechal Cândido Rondon, PR \\
\hline UEL pae 55 & H. glycines raça 3 & Espumoso, RS \\
\hline 831 (ESALQ) & Solo & Piracicaba, SP \\
\hline 832 (ESALQ) & Solenopsis invicta & Cuiabá, MT \\
\hline CG 266 & Solo & Balsas, MA \\
\hline CG 402 & Solo & Santarém, PA \\
\hline CG 184 & Solo & Rondonópolis, MT \\
\hline CG 175 & Meloidogyne sp. & Castanhal, PA \\
\hline
\end{tabular}

Ciência Rural, v.36, n.4, jul-ago, 2006. 
durante 25 dias a $25^{\circ} \mathrm{C}$ em meio BDA, sob regime alternado de 12 horas de luz. Após a inoculação, o substrato de arroz colonizado com o fungo foi incubado por 15 dias a $25^{\circ} \mathrm{C}$, no escuro. Decorrido esse período, determinou-se o número de esporos $\mathrm{g}^{-1}$ de arroz em câmara de Neubauer sob microscópio ótico.

O inóculo de $M$. paranaensis foi obtido por meio da multiplicação em raízes de tomateiros (Lycopersicon esculentum L.) cV. "Santa Clara" cultivados em vasos de barro durante 90 dias, em casade-vegetação, a partir das quais procedeu-se a extração dos ovos, segundo metodologia de BONETI \& FERRAZ (1981), obtendo-se uma suspensão ajustada para 1000 ovos e eventuais juvenis $\mathrm{mL}^{-1}$.

Para a determinação da eficiência dos isolados de $\boldsymbol{P}$. lilacinus, as plântulas de tomateiro cv. "Santa Clara" (uma por vaso) com 16 dias de idade foram transplantadas para vasos de plástico de 1,5L, contendo como substrato, solo e areia lavada previamente tratados com brometo de metila $\left(150 \mathrm{~mL} \mathrm{~m}^{-3}\right)$, mais esterco de curral, sendo este esterilizado em autoclave a $120^{\circ} \mathrm{C}$ por quatro horas, na proporção de 1:1:1 ( $\left.\mathrm{v} \mathrm{v}^{-1}\right)$. Aos 15 dias do transplantio, as plântulas foram inoculadas na região da rizosfera com $5 \mathrm{~mL}$ da suspensão contendo \pm 5000 ovos e eventuais juvenis de $\boldsymbol{M}$. paranensis, com o auxílio de pipeta esterilizada.

Em seguida, as plantas foram mantidas durante 45 dias para permitir a produção da segunda geração de ovos, uma vez que $\boldsymbol{P}$. lilacinus tem preferência pelo parasitismo destes. Decorrido esse período, procedeu-se ao corte das plantas na região do colo, à trituração do sistema radicular e à incorporação deste ao solo do mesmo vaso. $\mathrm{Na}$ seqüência, efetuou-se o transplantio de novas mudas de tomateiro do mesmo cultivar, na proporção de uma por vaso. Em seguida, procedeu-se à inoculação dos isolados de $\boldsymbol{P}$. lilacinus por meio da incorporação, em cada vaso, de $50 \mathrm{~g}$ de arroz colonizado ( $10^{9}$ esporos do fungo $\mathrm{g}^{-1}$ de arroz). Na testemunha, foram introduzidos apenas $50 \mathrm{~g}$ de arroz não colonizado. As práticas de adubação, irrigação e tratos culturais foram realizadas de acordo com as recomendações para a cultura.

Aos 45 dias da inoculação do fungo, foram avaliados o número de galhas, o número de massa de ovos, o número de ovos por sistema radicular e o peso da matéria fresca da parte aérea e do sistema radicular. Para a determinação dos números de galhas e de massa de ovos, as raízes foram lavadas em água corrente e imersas em solução de floxina B (15 $\mathrm{mg} \mathrm{L}^{-1}$ de água) durante 15 minutos para facilitar a visualização das massas de ovos e a contagem de galhas. Posteriormente, as raízes foram processadas, empregando-se a técnica de BONETI \& FERRAZ (1981) para extração e contagem dos ovos.
Para determinação da sobrevivência dos isolados de $\boldsymbol{P}$. lilacinus no solo, empregou-se a técnica da diluição seriada obtida a partir de $10 \mathrm{~g}$ de solo, em cinco repetições. Para cada amostra, procedeu-se à diluição seriada e, da diluição $10^{-3}$, foram retiradas alíquotas de $0,1 \mathrm{~mL}$, que foram distribuídas em placas de Petri contendo meio semi-seletivo de ALVES et al. (1998) modificado, em cinco repetições por amostra. No sexto dia da incubação a $25^{\circ} \mathrm{C}$ em B.O.D., foi determinado o número de colônias por placa e calculado o número de unidades formadoras de colônias (UFC) por grama de solo. Para isso, multiplicou-se a média de três repetições do número de colônias por placa por 10 , pois temos numa placa o número correspondente a 0,1mL. Assim, temos o número de microrganismos por $\mathrm{mL}$ da diluição plaqueada. A seguir, multiplica-se pela diluição. Os dados foram submetidos às análises de variância e do teste de Scott Knott a 5\% de probabilidade para comparação de médias.

\section{RESULTADOS E DISCUSSÃO}

Todos os isolados de $\boldsymbol{P}$. lilacinus testados promoveram a redução da população de $\boldsymbol{M}$. paranaensis nas raízes de tomateiro, quando comparados com a testemunha não tratada (Tabela 2). Resultados semelhantes foram verificados por FELLI et al. (1985), CABANILLAS et al. (1989a) e FREITAS et al. (1999), trabalhando com as espécies $\boldsymbol{M}$. incognita e M. javanica em tomateiros tratados com isolados de $\boldsymbol{P}$. lilacinus de diferentes locais de origem.

Para o número de galhas, as maiores reduções foram observadas com os isolados UEL pae 05, 08, 09, 13, 20, 21, 38, 41, 44, 54, ESALQ 831 e 832, diferindo dos demais tratamentos com percentuais de redução que variaram de 99,81 a 98,35\% em relação à testemunha (Tabela 2). Com exceção dos isolados ESALQ 831 e 832, UEL pae 05, 38, 44 e 54 (isolados de solo não identificado, de Solenopsis invicta, de mata nativa, quiabeiro, amoreira e de solo contaminado com H. glycines, respectivamente), os demais foram obtidos de solo cultivado com gramíneas. Estes resultados concordam com os encontrados por JATALA (1985), o qual observou que $\boldsymbol{P}$. lilacinus foi capaz de reduzir o número de galhas de $\boldsymbol{M}$. incognita em raízes de tomateiro. Esta diminuição pode ter sido motivada pelo parasitismo preferencial de ovos pelo fungo, ocasionando a morte dos embriões, o que resultou em menor número de juvenis infectantes (JATALA, 1986).

Os isolados UEL pae 05, 08, 09, 13, 20, 21, 38, 41, 44, 54, ESALQ 831 e 832 também reduziram significativamente o número de massa de ovos em percentuais que variaram de 99,70 a 98,52\% (Tabela 2). 
Seleção de isolados de Paecilomyces lilacinus (Thom.) Samson para controle de Meloidogyne paranaensis em tomateiro. 1059

Tabela 2 - Número de galhas, de ovos e massa de ovos de $\boldsymbol{M}$. paranaensis em raízes de tomateiro cv. "Santa Clara” inoculadas com $\boldsymbol{P}$. lilacinus.

\begin{tabular}{|c|c|c|c|c|c|c|c|c|c|}
\hline P. lilacinus & $\begin{array}{l}\text { Número de } \\
\text { galhas }\end{array}$ & & redução ${ }^{3}$ \% & $\begin{array}{l}\text { Número de } \\
\text { Massa ovos }\end{array}$ & & redução ${ }^{3}$ \% & Número de ovos & & redução ${ }^{3} \%$ \\
\hline UEL pae 01 & $11,11^{1}$ & $\mathrm{~d}$ & 84,26 & 8,53 & c & 84,57 & 165,10 & $\mathrm{~d}$ & 82,62 \\
\hline UEL pae 02 & 14,52 & C & 73,03 & 11,03 & $\mathrm{~b}$ & 74,45 & 210,13 & C & 71,74 \\
\hline UEL pae 03 & 11,37 & $\mathrm{~d}$ & 83,69 & 8,70 & c & 84,16 & 169,15 & d & 81,89 \\
\hline UEL pae 05 & 1,84 & $\mathrm{f}$ & 99,62 & 1,42 & e & 99,68 & 18,93 & $\mathrm{~h}$ & 99,75 \\
\hline UEL pae 06 & 17,02 & $\mathrm{~b}$ & 62,33 & 13,00 & $\mathrm{~b}$ & 63,96 & 244,40 & $\mathrm{~b}$ & 61,50 \\
\hline UEL pae 08 & 2,93 & $\mathrm{f}$ & 98,96 & 2,20 & e & 99,08 & 42,36 & g & 98,84 \\
\hline UEL pae 09 & 2,52 & $\mathrm{f}$ & 99,14 & 1,98 & e & 99,20 & 35,70 & g & 99,06 \\
\hline UEL pae 12 & 9,81 & $\mathrm{~d}$ & 87,75 & 7,50 & c & 88,18 & 145,25 & d & 86,60 \\
\hline UEL pae 13 & 1,75 & $\mathrm{f}$ & 99,66 & 1,51 & e & 99,61 & 10,06 & $\mathrm{~h}$ & 99,93 \\
\hline UEL pae 15 & 15,42 & $\mathrm{~b}$ & 67,82 & 11,74 & $\mathrm{~b}$ & 69,74 & 209,19 & c & 71,52 \\
\hline UEL pae 17 & 15,51 & $\mathrm{~b}$ & 65,49 & 11,93 & $\mathrm{~b}$ & 66,08 & 226,55 & $\mathrm{~b}$ & 63,86 \\
\hline UEL pae 18 & 13,42 & $\mathrm{C}$ & 76,39 & 10,27 & $\mathrm{~b}$ & 77,16 & 193,45 & C & 75,76 \\
\hline UEL pae 19 & 11,03 & $\mathrm{~d}$ & 84,44 & 8,47 & c & 84,90 & 164,09 & d & 82,75 \\
\hline UEL pae 20 & 1,90 & $\mathrm{f}$ & 99,64 & 1,43 & e & 99,67 & 19,84 & $\mathrm{~h}$ & 99,67 \\
\hline UEL pae 21 & 1,40 & $\mathrm{f}$ & 99,81 & 1,37 & e & 99,70 & 12,36 & $\mathrm{~h}$ & 99,89 \\
\hline UEL pae 22 & 4,59 & $\mathrm{e}$ & 97,37 & 3,40 & d & 97,67 & 67,73 & $\mathrm{f}$ & 97,06 \\
\hline UEL pae 23 & 13,44 & $\mathrm{C}$ & 77,08 & 10,32 & $\mathrm{~b}$ & 77,63 & 199,23 & c & 74,71 \\
\hline UEL pae 24 & 11,07 & $\mathrm{~d}$ & 84,34 & 8,48 & c & 84,72 & 163,87 & d & 82,99 \\
\hline UEL pae 38 & 1,85 & $\mathrm{f}$ & 99,62 & 1,46 & e & 99,64 & 20,65 & $\mathrm{~h}$ & 99,72 \\
\hline UEL pae 39 & 9,13 & $\mathrm{~d}$ & 89,37 & 6,98 & c & 89,75 & 135,14 & d & 88,39 \\
\hline UEL pae 41 & 1,59 & $\mathrm{f}$ & 99,73 & 1,35 & e & 99,70 & 12,63 & $\mathrm{~h}$ & 99,88 \\
\hline UEL pae 42 & 17,62 & $\mathrm{~b}$ & 59,75 & 11,64 & $\mathrm{~b}$ & 67,29 & 262,90 & $\mathrm{~b}$ & 54,98 \\
\hline UEL pae 44 & 3,57 & $\mathrm{f}$ & 98,35 & 2,65 & e & 98,52 & 50,66 & g & 98,32 \\
\hline UEL pae 45 & 7,90 & e & 90,61 & 6,10 & d & 90,63 & 114,00 & e & 90,79 \\
\hline UEL pae 47 & 4,78 & e & 97,10 & 3,67 & d & 97,19 & 70,87 & $\mathrm{f}$ & 96,74 \\
\hline UEL pae 48 & 7,38 & e & 93,16 & 5,64 & d & 93,44 & 110,72 & e & 92,09 \\
\hline UEL pae 49 & 10,80 & $\mathrm{~d}$ & 85,10 & 8,25 & c & 85,58 & 159,83 & d & 83,76 \\
\hline UEL pae 51 & 5,77 & $\mathrm{e}$ & 95,74 & 4,36 & d & 96,01 & 84,88 & $\mathrm{f}$ & 95,37 \\
\hline UEL pae 52 & 11,17 & $\mathrm{~d}$ & 84,06 & 8,51 & c & 15,18 & 166,87 & d & 81,97 \\
\hline UEL pae 54 & 1,74 & $\mathrm{f}$ & 99,64 & 1,34 & e & 99,67 & 17,13 & $\mathrm{~h}$ & 99,80 \\
\hline UEL pae 55 & 10,90 & $\mathrm{~d}$ & 84,74 & 8,28 & C & 85,58 & 161,50 & d & 83,33 \\
\hline 831 (ESALQ) & 2,86 & $\mathrm{f}$ & 98,55 & 2,19 & e & 99,08 & 40,82 & g & 98,92 \\
\hline 832 (ESALQ) & 1,46 & $\mathrm{f}$ & 99,78 & 1,33 & e & 99,64 & 11,40 & $\mathrm{~h}$ & 99,91 \\
\hline CG 266 & 10,77 & $\mathrm{~d}$ & 85,29 & 8,19 & C & 85,99 & 160,28 & d & 83,66 \\
\hline CG 402 & 6,69 & e & 94,27 & 5,13 & d & 94,41 & 99,38 & e & 93,60 \\
\hline CG 184 & 9,19 & $\mathrm{~d}$ & 89,02 & 7,04 & c & 89,42 & 135,55 & d & 88,13 \\
\hline CG 175 & 13,12 & C & 77,27 & 10,05 & $\mathrm{~b}$ & 77,99 & 194,37 & C & 75,28 \\
\hline Testemunha & 27,24 & $\mathrm{a}$ & - & 21,08 & $\mathrm{a}$ & - & 390,73 & $\mathrm{a}$ & - \\
\hline CV (\%) & 29,83 & & & 32,63 & & & 23,65 & & \\
\hline
\end{tabular}

${ }^{1}$ Dados transformados em v(x $\left.+0,5\right)$ para análise estatística. ${ }^{2}$ Médias não seguidas de mesma letra na coluna diferem entre si pelo teste de Scott Knott a $5 \%$ de probabilidade de erro. ${ }^{3}$ Calculado em relação à testemunha não inoculada com o fungo e referente aos dados originais. 
A maioria destes isolados foi obtida a partir de solo sob cultivo com gramíneas, à exceção dos isolados UEL pae 54, 38 e 44, ESALQ 831 e 832 . O fato de as fêmeas adultas de Meloidogyne (família Heteroderidae) concentrarem seus ovos em uma matriz gelatinosa pode facilitar o desenvolvimento do P. lilacinus e o parasitismo dos ovos. Segundo JATALA (1986), fungos parasitas de ovos são mais eficientes na redução da população de nematóides em comparação com aqueles que atuam como endoparasitas e predadores. Também são considerados os agentes mais promissores para o biocontrole de nematóides, uma vez que impedem ou reduzem a formação de ovos (KERRY et al., 1982).

Para o número de ovos por sistema radicular, os maiores percentuais de redução ocorreram nos tratamentos com UEL pae 05, 13, 20, 21, 38, 41, 54 e ESALQ 831 (Tabela 2). Dentre estes, UEL pae 13, 20, 21 e 41 foram obtidos de solo sob cultivo com gramíneas. Esses percentuais variaram entre 99,93 e 99,67\%. Os resultados obtidos no presente estudo estão de acordo com os obtidos por GODOY et al. (1982); VILLANUEVA \& DAVIDE (1984) e CARNEIRO \& GOMES (1993), os quais demonstraram que isolados de uma mesma espécie de fungo parasita de ovos procedentes de diferentes regiões geográficas variam quanto à capacidade parasitária. FREITAS et al. (1995), em avaliações sobre a eficiência de isolados de $\boldsymbol{P}$. lilacinus brasileiros quanto ao parasitismo de ovos de $\boldsymbol{M}$. javanica, observaram variações de 2 a $69 \%$ de ovos parasitados.

Apesar da capacidade dos isolados de $\boldsymbol{P}$.

lilacinus em reduzir a reprodução do nematóide, para nenhum deles foi observada a supressão total de $\boldsymbol{M}$. paranaensis nas raízes de tomateiro. CABANILLAS et al. (1989b) também não obtiveram a supressão de $\boldsymbol{M}$. ingognita em tomateiro com a adição de $\boldsymbol{P}$. lilacinus ao solo. No biocontrole de nematóides, não se pode esperar que os fungos tenham ação tão rápida ou efetiva quanto a do tratamento químico (FERRAZ \& SANTOS, 1995). Fungos parasitas de ovos sob condições de elevada infestação diferem de outros agentes de biocontrole, uma vez que seu efeito não é observado na primeira geração do nematóide, mas nas gerações seguintes (CARNEIRO, 1986); o que sugere a aplicação do biocontrolador de forma preventiva.

O peso médio de raízes de tomateiro variou para os diferentes tratamentos (Tabela 3), sendo observado o maior percentual de aumento para UEL pae 49, seguido dos isolados UEL pae 02, 13 e 42. Para o peso da matéria fresca da parte aérea, apenas os isolados UEL pae 55, 49 e 15 diferiram da testemunha não tratada, com aumentos de 31,57\%, 29,12\% e
$18,15 \%$, respectivamente. Estes aumentos indicam que a redução da população de nematóides nas raízes em função do tratamento com o fungo pode favorecer o desenvolvimento da planta. No entanto, no presente estudo, não foi observada correlação entre os tratamentos com P. lilacinus e o crescimento do tomateiro. DIAS \& FERRAZ (1994) também não observaram aumentos significativos no peso seco da parte aérea de tomateiros inoculados com $\boldsymbol{M}$. incognita e com o fungo antagonista Arthrobotrys spp., quando comparados com aqueles inoculados apenas com o nematóide, mesmo encontrando reduções significativas da população do nematóide. Estudos mostram ainda que nem sempre ocorrem aumentos na produção de plantas inoculadas com $\boldsymbol{P}$. lilacinus e infestadas por nematóides (JATALA, 1985).

A sobrevivência de $\boldsymbol{P}$. lilacinus no solo foi confirmada neste estudo, sendo os maiores números de colônias por placa e UFCs observados para os isolados CG 266, UEL pae 06, 24, CG 402, UEL pae 55, 52, CG 184, ESALQ 832, UEL pae 45, CG 175 e UEL pae 21, respectivamente (Tabela 4). Estes resultados são concordantes com os obtidos por GODOY et al. (1983), que verificaram a sobrevivência de $\boldsymbol{P}$. lilacinus no solo infestado com $\boldsymbol{M}$. arenaria, ao final do experimento. $\mathrm{O}$ parasitismo de ovos por fungos representa importante fenômeno biológico. Segundo TRIBE (1980), essa categoria normalmente consiste de fungos saprofíticos, que podem sobreviver independentemente da presença de ovos dos nematóides no solo. Por conseguinte, a importância desses microrganismos para o controle biológico é considerável.

A alta capacidade dos isolados de $\boldsymbol{P}$. lilacinus para reduzir a infecção por M. paranaensis, aliada à sobrevivência no solo, são fatores importantes a serem considerados na seleção de agentes de biocontrole. Dessa forma, o emprego de $\boldsymbol{P}$. lilacinus pode ser recomendado em áreas infestadas, buscando restabelecer o equilíbrio natural no solo e, assim, aumentar o efeito supressivo sobre os nematóides.

\section{CONCLUSÃO}

Isolados do fungo Paecilomyces lilacinus UEL pae 05, 08, 09, 13, 20, 21, 38, 41, 44, 54, 831 e 832 (ESALQ) foram considerados os melhores para o controle de $M$. paranaensis no cultivo de tomateiro em casa-de-vegetação, pois reduziram a população dos nematóides e apresentaram uma elevada taxa de sobrevivência no solo, características desejáveis para um agente de biocontrole. 
Tabela 3 - Peso médio de raízes e parte aérea de plantas de tomateiro cv. "Santa Clara” inoculadas com M. paranaensis e $\boldsymbol{P}$. lilacinus. Média de sete repetições em casa-de-vegetação.

\begin{tabular}{|c|c|c|c|c|c|c|c|}
\hline \multirow{2}{*}{$\begin{array}{l}\text { Isolados } \boldsymbol{P} \text {. lilacinus } \\
\text { UEL pae } 01\end{array}$} & \multirow{2}{*}{$\begin{array}{l}\text { Hospedeiro ou substrato } \\
\text { Solo (mata nativa) }\end{array}$} & \multicolumn{2}{|c|}{ Raízes (g) } & \multirow{2}{*}{$\begin{array}{c}\text { Aumento }^{2} \% \\
-19,82\end{array}$} & \multicolumn{2}{|c|}{ parte aérea (g) } & \multirow{2}{*}{$\begin{array}{c}\text { Aumento } \% \\
-21,86\end{array}$} \\
\hline & & 24,06 & $\mathrm{e}^{1}$ & & 21,09 & $\mathrm{c}$ & \\
\hline UEL pae 02 & Solo (café) & 62,45 & $\mathrm{~b}$ & 108,10 & 29,53 & $\mathrm{~b}$ & 9,41 \\
\hline UEL pae 03 & Solo (trigo) & 49,68 & c & 65,54 & 30,34 & $\mathrm{~b}$ & 12,41 \\
\hline UEL pae 05 & Solo (mata nativa) & 48,99 & $\mathrm{C}$ & 63,25 & 27,23 & $\mathrm{~b}$ & 0,89 \\
\hline UEL pae 06 & Solo (pastagem) & 38,44 & $\mathrm{~d}$ & 28,09 & 24,87 & $\mathrm{~b}$ & $-7,85$ \\
\hline UEL pae 08 & Solo (trigo) & 20,02 & e & $-33,29$ & 16,57 & $\mathrm{~d}$ & $-38,61$ \\
\hline UEL pae 09 & Solo (milho) & 17,66 & e & $-41,15$ & 17,33 & $\mathrm{~d}$ & $-35,79$ \\
\hline UEL pae 12 & Solo (Pinus sp.) & 46,26 & c & 54,15 & 30,07 & $\mathrm{~b}$ & 11,41 \\
\hline UEL pae 13 & Solo (pastagem) & 60,17 & $\mathrm{~b}$ & 100,50 & 27,52 & $\mathrm{~b}$ & 1,96 \\
\hline UEL pae 15 & Solo (milho) & 34,26 & $\mathrm{~d}$ & 14,16 & 31,89 & $\mathrm{a}$ & 18,15 \\
\hline UEL pae 17 & Solo (pastagem) & 33,54 & $\mathrm{~d}$ & 11,76 & 27,52 & $\mathrm{~b}$ & 1,96 \\
\hline UEL pae 18 & Solo (pastagem) & 31,30 & $\mathrm{~d}$ & 4,30 & 24,58 & $\mathrm{~b}$ & $-8,93$ \\
\hline UEL pae 19 & Solo (cana-de-açúcar) & 22,23 & e & $-25,92$ & 17,09 & $\mathrm{~d}$ & $-36,68$ \\
\hline UEL pae 20 & Solo (milho) & 12,94 & e & $-56,88$ & 16,72 & $\mathrm{~d}$ & $-38,05$ \\
\hline UEL pae 21 & Solo (trigo) & 16,05 & $\mathrm{e}$ & $-46,52$ & 17,42 & $\mathrm{~d}$ & $-35,46$ \\
\hline UEL pae 22 & Solo (mata nativa) & 18,36 & $\mathrm{e}$ & $-38,82$ & 16,07 & $\mathrm{~d}$ & $-40,46$ \\
\hline UEL pae 23 & Solo (café) & 32,40 & $\mathrm{~d}$ & 7,96 & 18,09 & $\mathrm{~d}$ & $-32,98$ \\
\hline UEL pae 24 & Solo (trigo) & 43,34 & $\mathrm{C}$ & 44,42 & 21,70 & $\mathrm{C}$ & $-19,60$ \\
\hline UEL pae 38 & Solo (quiabeiro) & 21,69 & $\mathrm{e}$ & $-27,72$ & 12,96 & e & $-51,98$ \\
\hline UEL pae 39 & Solo (milho) & 33,51 & $\mathrm{~d}$ & 11,66 & 29,36 & $\mathrm{~b}$ & 8,78 \\
\hline UEL pae 41 & Solo (pastagem) & 25,80 & $\mathrm{e}$ & $-14,03$ & 21,83 & $\mathrm{c}$ & $-19,12$ \\
\hline UEL pae 42 & Solo (café) & 55,75 & $\mathrm{~b}$ & 85,77 & 28,85 & $\mathrm{~b}$ & 6,89 \\
\hline UEL pae 44 & Solo (amora) & 21,21 & $\mathrm{e}$ & $-29,32$ & 21,74 & $\mathrm{C}$ & $-19,45$ \\
\hline UEL pae 45 & Solo (milho) & 38,26 & $\mathrm{~d}$ & 27,49 & 21,03 & $\mathrm{c}$ & $-22,08$ \\
\hline UEL pae 47 & Solo (café) & 23,50 & e & $-21,69$ & 13,58 & e & $-49,69$ \\
\hline UEL pae 48 & Solo (trigo) & 23,30 & $\mathrm{e}$ & $-22,36$ & 13,35 & $\mathrm{e}$ & $-50,54$ \\
\hline UEL pae 49 & M. javanica e $H$. glycines raça 3 & 88,99 & $\mathrm{a}$ & 196,53 & 34,85 & a & 29,12 \\
\hline UEL pae 51 & M. incognita e H. glycines & 24,06 & $\mathrm{e}$ & $-19,83$ & 14,85 & $\mathrm{e}$ & $-44,98$ \\
\hline UEL pae 52 & M. incognita e H. glycines & 20,88 & $\mathrm{e}$ & $-30,42$ & 15,12 & $\mathrm{e}$ & $-43,98$ \\
\hline UEL pae 54 & H. glycines & 20,24 & $\mathrm{e}$ & $-32,56$ & 17,55 & $\mathrm{~d}$ & $-34,98$ \\
\hline UEL pae 55 & H. glycines raça 3 & 52,84 & $\mathrm{C}$ & 76,07 & 35,51 & $\mathrm{a}$ & 31,57 \\
\hline 831 (ESALQ) & Solo & 38,09 & $\mathrm{~d}$ & 26,92 & 27,08 & $\mathrm{~b}$ & 0,33 \\
\hline 832 (ESALQ) & Solenopsis invicta & 25,34 & e & $-15,56$ & 16,59 & $\mathrm{~d}$ & $-38,53$ \\
\hline CG 266 & Solo & 20,42 & $\mathrm{e}$ & $-31,96$ & 15,70 & $\mathrm{~d}$ & $-41,83$ \\
\hline CG 402 & Solo & 26,79 & $\mathrm{e}$ & $-10,73$ & 18,06 & $\mathrm{~d}$ & $-33,09$ \\
\hline CG 184 & Solo & 25,21 & $\mathrm{e}$ & $-15,99$ & 14,53 & $\mathrm{e}$ & $-46,17$ \\
\hline CG 175 & Meloidogyne sp. & 23,11 & $\mathrm{e}$ & $-22,99$ & 14,04 & $\mathrm{e}$ & $-47,98$ \\
\hline Testemunha & & 30,01 & $\mathrm{~d}$ & - & 26,99 & $\mathrm{~b}$ & - \\
\hline CV (\%) & & 31,80 & & & 17,14 & & \\
\hline
\end{tabular}

${ }^{1}$ Médias não seguidas de mesma letra na coluna diferem entre si pelo teste de Scott Knott a $5 \%$ de probabilidade de erro. ${ }^{2}$ Calculado em relação à testemunha não inoculada com o fungo. 
Tabela 4 - Sobrevivência de isolados de P. lilacinus em solo cultivado com tomateiro cv. "Santa Clara" inoculado com M. Paranaensis expressa em número de colônias por placa e transformada em unidades formadoras de colônias (UFC) por grama de solo.

\begin{tabular}{|c|c|c|c|c|}
\hline \multirow{2}{*}{$\begin{array}{l}\text { Isolados } \boldsymbol{P} \text {. lilacinus } \\
\text { UEL pae } 01\end{array}$} & \multirow{2}{*}{$\begin{array}{l}\text { Hospedeiro ou substrato } \\
\text { Solo (mata nativa) }\end{array}$} & \multicolumn{2}{|c|}{$\mathrm{N}^{\circ}$ de colônias / placa } & \multirow{2}{*}{$\frac{\text { UFC / g solo }}{0,11 \times 10^{3}}$} \\
\hline & & $10,60^{1}$ & $\mathrm{f}^{2}$ & \\
\hline UEL pae 02 & Solo (café) & 97,20 & e & $0,97 \times 10^{3}$ \\
\hline UEL pae 03 & Solo (trigo) & 14,20 & $\mathrm{f}$ & $0,14 \times 10^{3}$ \\
\hline UEL pae 05 & Solo (mata nativa) & 161,00 & c & $1,61 \times 10^{3}$ \\
\hline UEL pae 06 & Solo (pastagem) & 225,40 & $\mathrm{a}$ & $2,25 \times 10^{3}$ \\
\hline UEL pae 08 & Solo (trigo) & 45,60 & $\mathrm{f}$ & $0,46 \times 10^{3}$ \\
\hline UEL pae 09 & Solo (milho) & 72,20 & e & $0,72 \times 10^{3}$ \\
\hline UEL pae 12 & Solo (Pinus sp.) & 180,40 & $\mathrm{~b}$ & $1,80 \times 10^{3}$ \\
\hline UEL pae 13 & Solo (pastagem) & 12,40 & $\mathrm{f}$ & $0,12 \times 10^{3}$ \\
\hline UEL pae 15 & Solo (milho) & 13,60 & $\mathrm{f}$ & $0,14 \times 10^{3}$ \\
\hline UEL pae 17 & Solo (pastagem) & 181,20 & $\mathrm{~b}$ & $1,81 \times 10^{3}$ \\
\hline UEL pae 18 & Solo (pastagem) & 12,80 & $\mathrm{f}$ & $0,13 \times 10^{3}$ \\
\hline UEL pae 19 & Solo (cana-de-açúcar) & 11,80 & $\mathrm{f}$ & $0,12 \times 10^{3}$ \\
\hline UEL pae 20 & Solo (milho) & 184,20 & $\mathrm{~b}$ & $1,84 \times 10^{3}$ \\
\hline UEL pae 21 & Solo (trigo) & 201,00 & a & $2,01 \times 10^{3}$ \\
\hline UEL pae 22 & Solo (mata nativa) & 152,80 & c & $1,53 \times 10^{3}$ \\
\hline UEL pae 23 & Solo (café) & 162,60 & c & $1,63 \times 10^{3}$ \\
\hline UEL pae 24 & Solo (trigo) & 213,60 & $\mathrm{a}$ & $2,14 \times 10^{3}$ \\
\hline UEL pae 38 & Solo (quiabeiro) & 196,20 & $\mathrm{~b}$ & $1,96 \times 10^{3}$ \\
\hline UEL pae 39 & Solo (milho) & 9,00 & $\mathrm{f}$ & $0,09 \times 10^{3}$ \\
\hline UEL pae 41 & Solo (pastagem) & 12,60 & $\mathrm{f}$ & $0,13 \times 10^{3}$ \\
\hline UEL pae 42 & Solo (café) & 5,00 & $\mathrm{f}$ & $0,05 \times 10^{3}$ \\
\hline UEL pae 44 & Solo (amora) & 7,20 & $\mathrm{f}$ & $0,07 \times 10^{3}$ \\
\hline UEL pae 45 & Solo (milho) & 203,80 & a & $2,04 \times 10^{3}$ \\
\hline UEL pae 47 & Solo (café) & 104,80 & e & $1,05 \times 10^{3}$ \\
\hline UEL pae 48 & Solo (trigo) & 10,60 & $\mathrm{f}$ & $0,11 \times 10^{3}$ \\
\hline UEL pae 49 & M. javanica e H. glycines raça 3 & 133,80 & $\mathrm{~d}$ & $1,34 \times 10^{3}$ \\
\hline UEL pae 51 & M. incognita e H. glycines & 120,80 & d & $1,21 \times 10^{3}$ \\
\hline UEL pae 52 & M. incognita e H. glycines & 206,20 & a & $2,06 \times 10^{3}$ \\
\hline UEL pae 54 & H. glycines & 110,40 & d & $1,10 \times 10^{3}$ \\
\hline UEL pae 55 & H. glycines raça 3 & 207,40 & a & $2,07 \times 10^{3}$ \\
\hline 831 (ESALQ) & Solo & 95,60 & e & $0,96 \times 10^{3}$ \\
\hline 832 (ESALQ) & Solenopsis invicta & 205,20 & a & $2,05 \times 10^{3}$ \\
\hline CG 266 & Solo & 227,60 & a & $2,28 \times 10^{3}$ \\
\hline CG 402 & Solo & 207,60 & a & $2,08 \times 10^{3}$ \\
\hline CG 184 & Solo & 205,40 & $\mathrm{a}$ & $2,05 \times 10^{3}$ \\
\hline CG 175 & Meloidogyne sp. & 201,20 & a & $2,01 \times 10^{3}$ \\
\hline Testemunha & & 0,00 & $\mathrm{f}$ & $0,00 \times 10^{3}$ \\
\hline CV (\%) & & 18,13 & & \\
\hline
\end{tabular}

${ }^{1}$ Médias da contagem em 25 repetições (5 placas x 5 vasos). ${ }^{2}$ Médias não seguidas de mesma letra na coluna diferem entre si pelo teste de Scott Knott a 5\% de probabilidade de erro. "Média de três repetições do número de colônias por placa (placas com 0,1mL da diluição $10^{-3}$ ) x $10 \times 10^{-3}$. 


\section{AGRADECIMENTOS E APRESENTAÇÃO}

Parte da Tese de Doutorado do primeiro autor. Apoio: Coordenação de Aperfeiçoamento de Pessoal de Nível Superior (Capes) e Programa de Pós-graduação em Agronomia - UEL.

\section{REFERÊNCIAS}

ALVES, S.B. et al. Técnicas de laboratório. In: ALVES, S.B (Ed). Controle microbiano de insetos. Piracicaba: Fundação de Estudos Agrários “Luiz de Queiroz”, 1998. p.637-711.

BONETI, J.I.S.; FERRAZ, S. Modificação do método de Hussey \& Barker para a extração de ovos de Meloidogyne exigua em cafeeiro. Fitopatologia Brasileira, v.6, n.3, p.553, 1981.

CABANILLAS, E. et al. Growth of isolates of Paecilomyces lilacinus on their efficacy in biocontrol of Meloidogyne incognita on tomato. Journal of Nematology, Lakeland, v.21, n.2, p.164-172, 1989a.

CABANILLAS, E. et al. Survival of Paecilomyces lilacinus in selected carriers and related effects on Meloidogyne incognita on tomato. Journal of Nematology, v.21, n.1, p.121-130, 1989b.

CARNEIRO, R.M.D.G. Estude des Possibilities D'utilisation du Champignon Nematophage Paecilomyces lilacinus (Thom.) Samson, 1974, Comme Agent de Lutte Biologique contre Meloidogyne arenaria (Neal, 1889), Chitwood, 1949. 1986. 119f. Tese (Doutorado) - Cours do Pos Graduation in Parasitologie, Academie de Montpellier. Universite des Sciences et Techniques du Languedoc, France.

CARNEIRO, R.M.D.G. Princípios e tendências do controle biológico de nematóides com fungos nematófagos. Pesquisa Agropecuária Brasileira, v.27, p.113-121, 1992.

CARNEIRO, R.M.D.G.; GOMES, C.B. Metodologia e teste de patogenicidade de Paecilomyces lilacinus e $\boldsymbol{P}$. fumosoroseus em ovos de Meloidogyne javanica. Nematologia Brasileira, v.7, n.1, p. 66-75, 1993

CARNEIRO, R.M.D.G.; CAYROL, J.C. Relationship between inoculum density of the nematophagus fungus Paecilomyces lilacinus and control of Meloidogyne arenaria on tomato. Revue Nematologie, v.14, n.4, p.629-634, 1991.

COSTA, S.B. et al. Fungos associados a cistos de Heterodera glycines no Brasil. Nematologia Brasileira, v.21, n.2, p.3137, 1997.

COSTA, S.B.; CAMPOS, V.P. Production of Heterodera glycines females in hydroponic solution and pathogenicity tests with fungi isolated from cysts to $\boldsymbol{H}$. glycines and Meloidogyne spp. females. Summa Phytopathologica, v.23, n. 3-4, p.239243, 1997.

DIAS, W.P.; FERRAZ, S. Avaliação de espécies de Arthrobotrys para o controle de Meloidogyne incognita. Fitopatologia Brasileira, v.19, n.2, p.189-193, 1994.

DOMSCH, K.H. et al. Compendium of soil fungi. New York: Academic, 1980. v.1. 859p.
DUNCAN, L.W. Current options for nematode management. Annual Review of Phytopathology, v. 29, p.469-490, 1991.

DUNN, M.T. et al. Colonization of nematode eggs by Paecilomyces lilacinus (Thom) Samson as observed with scanning electron microscope. Scanning Electron Microscopy, p.1351-1357, 1982.

FARIA, M.R.; TIGANO, M.S. Coleção de fungos entomopatogênicos do Cenargen. Brasília: Embrapa, Serviço de Produção e Informação, 1996. 76p.

FELLI, L.F.S. et al. Efeito de Paecilomyces lilacinus, carbamatos e matéria orgânica no controle de Meloidogyne incognita em tomateiro. Nematologia Brasileira, v.9, (único), p.34-35, 1985.

FERRAZ, S.; SANTOS, M.A. Controle biológico de fitonematóides pelo uso de fungos. In: LUZ, W.C. (Ed). Revisão Anual de Patologia de Plantas. Passo Fundo: EMBRAPA, 1995. p.283-314

FREITAS, L.G. et al. Effectiveness of different isolates of Paecilomyces lilacinus and an isolate of Cylindrocarpon destructans on the control of Meloidogyne javanica. Nematropica, v.25, n.2, p.109-115, 1995.

FREITAS, L.G. et al. Controle de Meloidogyne javanica em tomateiro pela produção de mudas e substrato infestado com Paecilomyces lilacinus. Nematologia Brasileira, v.23, n.1, p.65-71, 1999.

GODOY, G. et al. Parasitism of eggs of Heterodera glycines and Meloidogyne arenaria by fungi isolated from cyst of $\boldsymbol{H}$. glycines. Nematropica, v.12, n.1, p.111-119, 1982.

GODOY, G. et al. Fungal parasites of Meloidogyne arenaria eggs in Alabama soil. A mycological survey and greenhouse studies. Nematropica, v.13, p.207-213, 1983.

HEWLETT, T.E. et al. Evaluation of Paecilomyces lilacinus as a biocontrol agent of Meloidogyne javanica on tobacco. Journal of Nematology, v.20, n.4, p.578-584, 1988.

JATALA, P. Biological control of nematodes. In: SASSER, J.N.; CARTER, E.C. An advanced treatise on Meloidogyne. North Carolina: North Carolina State University, 1985. p.303308.

JATALA, P. Biological control of plant-parasitic nematodes. Annual Review of Phytopathology, v.24, p.453-489, 1986.

KERRY, B.R. An assessment of progress toward microbial controle of plant parasitic nematode. Journal of Nematology, v. 22, n.45, p.621-631, 1990. (Supplement)

KERRY, B.R. et al. Studies of the cereal-cyst nematode, Heterodera avenae Woll. under continuous cereals, 1975-1978. II. Fungal parasitism of nematode eggs and females. Annals Applied Biology, v.100, n.3, p.489-499, 1982.

MIZOBUTSI, E.H. et al. Avaliação do parasitismo de diversos isolados fúngicos em ovos de Heterodera glycines e Meloidogyne javanica. Nematologia Brasileira, 24, n.2, p.167-172, 2000. NOVARETTI, W.R.T. et al. Efeito da aplicação conjunta do fungo Paecilomyces lilacinus e do nematicida Furadan $5 \mathrm{G}$ no 
controle de nematóides em cana-de-açúcar. Nematologia Brasileira, v.X, p.133-144, 1986.

SANTOS, J.M. dos et al. Detection and ecology of nematophagous fungi from brazilian soils. Nematologia Brasileira, v.15, n.2, p.121-134, 1991

SOSA-GOMEZ, D.R. Fungos entomopatogênicos: catálogo de isolados. Londrina: Embrapa Soja, 2002. V.1, p.1-32. (Série Documentos).

STIRLING G.R. Biological control of plant parasitic nematodes. Wallingford: CAB International, 1991. 282p.
TIGANO-MILANI, M.S. et al. Ocorrência natural de Beauveria bassiana (Bals.) Vuill., Metarhizium anisopliae (Metsch.) Sorok. e Paecilomyces sp. em solos de diferentes regiões do Brasil. Anais da Sociedade Entomológica do Brasil, v.22, n.2, p.391-393, 1993.

TRIBE, H.T. Prospects for the control of plant parasitic nematodes. Parasitology, v.81, p.619-639, 1980.

VILLANUEVA, L.M.; DAVIDE, R.G. Evaluation of several isolates of soil fungi for biological control of root-knot nematodes. Philippine Agriculturist, v.67, n.4, p.361-371, 1984. 